\title{
Ultrasonographic evaluation of extracapsular vascular invasion for subcapsular nodules of the thyroid
}

\author{
Minghang Lin ${ }^{1,2 \#}$, Yiming Su ${ }^{3 \#}$, Yinan Huang ${ }^{4 \#}$, Huihao Zhang ${ }^{4 \#}$, Jinshu Zeng ${ }^{4}$, Yong Zhuang ${ }^{4}$, \\ Xiaojian Ye ${ }^{4}$, Shuqiang Chen ${ }^{4}$ \\ ${ }^{1}$ Department of Ultrasound, Fuqing City Hospital Affiliated of Fujian Medical University, Fuqing, China; ${ }^{2}$ Department of Ultrasound, First Affiliated \\ Hospital of Fujian Medical University, Fuzhou, China; ${ }^{3}$ Department of Ultrasound, First Affiliated Hospital of Xiamen University, Xiamen, China; \\ ${ }^{4}$ Department of Ultrasound, First Affiliated Hospital of Fujian Medical University, Fuzhou, China \\ Contributions: (I) Conception and design: S Chen; (II) Administrative support: S Chen; (III) Provision of study materials or patients: All authors; (IV) \\ Collection and assembly of data: M Lin, Y Su, S Chen, H Zhang, Y Huang; (V) Data analysis and interpretation: M Lin, Y Su, S Chen, H Zhang, Y \\ Huang; (VI) Manuscript writing: All authors; (VII) Final approval of manuscript: All authors. \\ \#These authors contributed equally to this work. \\ Correspondence to: Shuqiang Chen. Department of Ultrasound, First Affiliated Hospital of Fujian Medical University, No. 20, Chazhong Road, \\ Taijiang District, Fuzhou 350000, China. Email: Chenshu0518@163.com.
}

Background: Ultrasound provides a high-frequency spatial resolution. In this study, we used the combined pathological features of extrathyroid extension (ETE) measured by ultrasound to evaluate the vascular manifestations of subcapsular differentiated thyroid cancer. Our study aims to explore the value of highfrequency ultrasonography in the evaluation of extracapsular vascular invasion for the evaluation of both benign and malignant nodules and the prediction of ETE.

Methods: A total of 167 thyroid nodules were enrolled in this study. High-frequency ultrasonography was used to observe the relationship between the blood flow of the nodules and the capsules. The blood flow was divided into two types according to the relationship: non-extracapsular invasive blood flow and extracapsular invasive blood flow. Non-extracapsular blood flow was defined as any flow seen inside or around the nodule that did not extend beyond the thyroid gland. Extracapsular invasive blood flow was defined as any blood flow seen inside or around the nodule that flowed across the capsule and extended beyond the thyroid gland. A comparison of the different types of blood flow to judge the nature of thyroid nodules for predicting ETE was performed.

Results: Out of 167 nodules, 81 cases of nodules were the non-extracapsular invasive blood flow type, while the remaining 86 cases of nodules were classified as the extracapsular invasive blood flow type. Nodules with distinct types of blood flow were significantly different in malignancy rates between the nodules $(\mathrm{P}<0.001)$. The incidence rate of ETE was also significantly different between the malignant nodules with distinct types of blood flows.

Conclusions: Extracapsular vascular invasion is a good indicator for the evaluation of benign and malignant nodules. Using it as an indicator provides physicians with a potential tool for the prediction of ETE.

Keywords: Extrathyroid extension (ETE); blood flow; metastasis; ultrasonography; thyroid carcinoma

Submitted Feb 06, 2020. Accepted for publication Sep 10, 2020.

doi: $10.21037 /$ tcr-20-888

View this article at: http://dx.doi.org/10.21037/tcr-20-888 


\section{Introduction}

An extrathyroid extension (ETE) is an independent risk factor for the recurrence of differentiated thyroid cancer. The occurrence of this cancer in individuals can reduce the 10 -year survival rate from $99.3 \%$ to $63.0 \%$ (1). Research has found that ETE also significantly increases the metastasis rate of central lymph nodes and increases the risk of distant metastasis (2). Furthermore, it increases the risk of non-total thyroidectomy, which may lead to higher tumor recurrence rates and mortality (3). It is usually controversial on the extent of surgery when treating low-risk patients with thyroid cancer. The presence of ETE is a vital factor in deciding the extent of surgery when the cancer is small, because this is usually changed from lobectomy to total or near-total thyroidectomy if there is ETE. Also, the prognosis of thyroid carcinoma with ETE is worse than that without ETE (3). The occurrence and extent of ETE determine the stage of thyroid cancer. According to the thyroid cancer TNM staging system (8th edition), edited by the American Joint Committee on Cancer (AJCC) (4), any tumor with extrathyroidal infiltration is categorized as being in the T3 stage. Therefore, the presence of ETE has vital clinical significance as a perioperative assessment tool. At present, the use of ultrasound is the preferred and most effective method for thyroid tumor imaging. However, there is no convenient index for the ultrasonographic evaluation of ETE. Because of the complicated pathophysiological processes underlying the occurrence and development of malignant tumors, like the proliferation and invasion of blood vessels, technologies such as the Doppler ultrasound offer clear advantages in the evaluation of the pathophysiological changes of these subtle blood vessels. Extracapsular vascular invasion is usually described as a histological concept in pathological examination, which can be considered a form of ETE (5). However, this sign has not been further studied in ultrasound. This study aimed to find an ultrasonic sign of extracapsular vascular invasion to be used as a standard for predicting ETE. We used the advantages of the spatial resolution of high-frequency ultrasound to evaluate the vascular manifestations of subcapsular differentiated thyroid cancer combined with the pathological features of ETE of the thyroid nodules. We present the following article in accordance with the STROBE reporting checklist (available at http://dx.doi. org/10.21037/tcr-20-888).

\section{Methods}

\section{Participants}

One hundred and sixty-seven thyroid nodule specimens were contributed to this study from hospitalized patients who underwent preoperative thyroid ultrasonography between January 2015 and April 2018. All of the nodules were accompanied by postoperative pathological results.

Nodules that met the following criteria were included: (I) nodules adjacent to or in contact with the anterior thyroid capsule; (II) only a single nodule in the thyroid gland; and (III) A color Doppler showed there were blood flow signals inside or around the nodules.

The exclusion criteria for this study included: (I) the nodules were located in the isthmus; (II) the capsule destruction and disruption was caused by previous thyroid trauma or another non-tumor infiltration; (III) history of local ablation of thyroid nodules; or (IV) the patient had subacute thyroiditis or had previously undergone thyroid surgery.

The study was conducted in accordance with the Declaration of Helsinki (as revised in 2013). This retrospective study was approved by the Ethics Review Committee of the First Affiliated Hospital of Fujian Medical University $\{[2016] 117\}$; the Review board determined that informed consent was not needed from the enrolled patients.

\section{Instruments and methods}

We used the GE Logiq E9 (GE Healthcare, Waukesha, WI, USA) and Philips iU22 (Philips, Best, the Netherlands) ultrasound diagnostic apparatuses (shallow inverter probe, frequency 5-15 MHz). The ultrasound images of the subcapsular thyroid nodules were analyzed by two sonographers with more than 10 years of working experience and were blinded to the pathological findings of the nodules. The high-frequency ultrasounds were used to observe the location, size, shape, border, presence/ absence of halo, internal echo and calcification, blood flow inside or around the nodules, and enlarged lymph nodes in the bilateral neck. Pressure on the nodules was carefully avoided. A cross and longitudinal section ultrasonographic images were captured for each nodule. Next, the distribution of blood vessels inside or around the nodules and the relationships between the blood flow and the anterior thyroid capsule were observed. The blood 


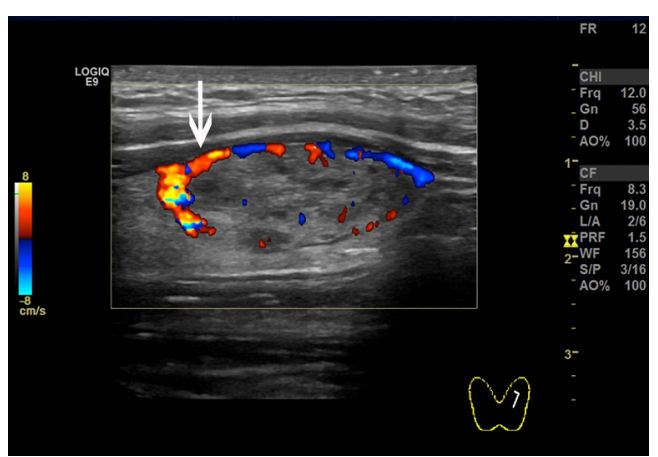

Figure 1 Non-extracapsular invasive blood flow. Blood flows are seen inside or around the nodule, and the blood flow does not extend beyond the thyroid gland (white arrow).

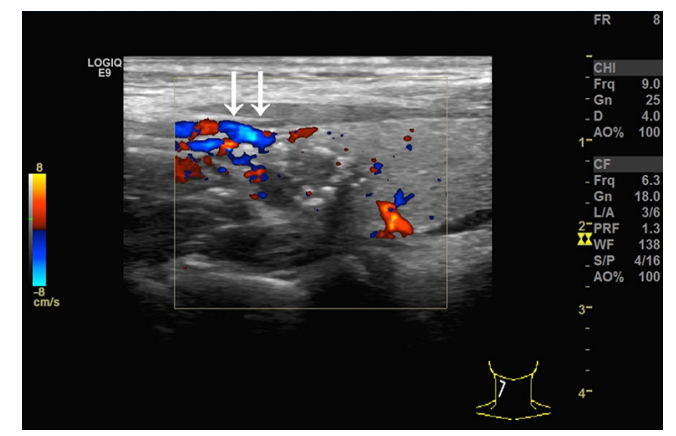

Figure 2 Extracapsular invasive blood flow. Blood flows are seen inside or around the nodule, and the blood flow crosses the capsule and extends beyond the thyroid gland (white arrows).

flows were then categorized into two classes according to their relationship. One, the non-extracapsular invasive blood flow category (Figure 1). Two, the extracapsular invasive blood flow category (Figure 2). Concerning the pathological findings of thyroid nodules, the predictive effects of the blood flow types on the diagnosis of nodules and extracapsular invasion were evaluated. If the results of the evaluation were inconsistent by the two examining physicians, the images were evaluated jointly, and the agreed-upon results were recorded.

\section{Statistical analysis}

Data analysis was performed using the SPSS statistical analysis software 17.0 (IBM, Armonk, NY, USA). Quantitative data were expressed as a percent of the total nodules, and the differences between the groups were analyzed using a chi-squared test. All measurements were represented by $\bar{x} \pm s$. For the variables with normal distribution and homogeneity of variance, independent sample t-tests were used for data analysis. A P value of $<0.05$ was considered statistically significant.

\section{Results}

\section{Patient clinical information}

One hundred and sixty-seven nodules were included in the study, including 83 cases of benign nodules and 84 cases of malignant nodules. There were 56 cases of nodular goiter (benign), 26 cases of thyroid adenomas, and one case of Hashimoto's thyroiditis among the benign nodules. There were 79 cases of papillary carcinoma, two cases of medullary carcinoma, three cases of follicular carcinoma, and no undifferentiated types among the malignant nodules.

\section{Correlations between ultrasound evaluation and nodule patbology}

The routine ultrasound assessment of the 167 nodules is shown in Table 1. Benign nodules and malignant nodules showed statistically significant differences in internal microcalcification, irregular nodule edges, aspect ratio, internal blood flow, and the presentation of the bilateral cervical lymph nodes. There were significant differences in the distribution of the two types of blood flow between the benign and malignant nodules $(\mathrm{P}<0.001)$. The incidence rate in the malignant nodules of the extracapsular invasive blood flow group (92.86\%) was much higher than the incidence rate in the non-extracapsular invasive blood flow group (7.41\%).

\section{Blood flow types and ETEs}

The incidence rate of malignant nodules was significantly different in the nodules with distinct types of blood flow. In the nodules with non-extracapsular invasive blood flow, the proportion of malignant nodules was only $7.41 \%$, while in the nodules with extracapsular invasive blood flow, the proportion of malignant nodules was $90.70 \%$ $(\mathrm{P}<0.001)$. The extracapsular invasion of malignant nodules with different blood flow types is shown in Table 2. The incidence rate of ETE with extracapsular invasive blood flow was also significantly higher than that of non-encapsulated invasion in malignant nodules $(\mathrm{P}<0.001)$. Extracapsular vascular invasion was characterized 
Table 1 Routine ultrasonographic evaluation of 167 benign and malignant nodules

\begin{tabular}{lccc}
\hline Variables & Benign nodules $(\mathrm{N}=83)$ & Malignant nodules $(\mathrm{N}=84)$ & $\mathrm{P}$ value \\
\hline Male & $13(15.66)$ & $17(20.24)$ & 0.441 \\
Age (years), mean $\pm \mathrm{SD}$ & $47.64 \pm 12.99$ & $46.68 \pm 11.02$ & 0.607 \\
Max-diameter $(\mathrm{cm})$, mean $\pm \mathrm{SD}$ & $1.45 \pm 0.58$ & $1.29 \pm 0.68$ & 0.114 \\
Irregular edges $(\mathrm{N}=68)$ & $8(9.64)$ & $60(71.43)$ & $<0.001$ \\
Aspect ratio $>1(\mathrm{~N}=38)$ & $5(6.02)$ & $33(39.29)$ & $<0.001$ \\
Micro-calcification $(\mathrm{N}=98)$ & $30(36.14)$ & $59(70.24)$ & $<0.001$ \\
Internal blood flow $(\mathrm{N}=99)$ & $40(48.19)$ & $19(22.62)$ & 0.004 \\
Peripheral lymph nodes $(\mathrm{N}=19)$ & $0(0)$ & $6(7.14)$ & $<0.001$ \\
Non-extracapsular invasion $(\mathrm{N}=81)$ & $75(90.36)$ & $78(92.86)$ & $<0.001$ \\
Extracapsular invasion $(\mathrm{N}=86)$ & $8(9.64)$ & $<0.001$ & $<$
\end{tabular}

Unless otherwise specified, the above data are presented as percent of nodules; Percentage represents the number of nodules/the number of benign or malignant; $\mathrm{P}<0.05$ was threshold for statistical significance between benign and malignant group.

Table 2 The comparison of pathological results and extracapsular invasion among the malignant nodules combined with different blood flow

\begin{tabular}{lccc}
\hline Variables & $\begin{array}{c}\text { Non-extracapsular } \\
\text { invasion }(\mathrm{N}=6)\end{array}$ & $\begin{array}{c}\text { Extracapsular } \\
\text { invasion }(\mathrm{N}=78)\end{array}$ & P value \\
\hline ETE & 0 & $76(97.44)$ & $<0.001$ \\
non-ETE & $6(100.00)$ & $2(2.56)$ & $<0.001$ \\
\hline
\end{tabular}

Unless otherwise specified, data are presented as percent of nodules; $P$ value $<0.05$ indicates statistically significant difference between the non-extracapsular vascular invasion and extracapsular vascular invasion. ETE, extrathyroid extension.

pathologically by neovascularization through the thyroid capsule, invading the extra-thyroid tissues (Figure 3).

\section{Discussion}

Several nerve bundles, capillaries, and lymphatic capillaries within the thyroid capsule make up the extrathyroidal tissues. Malignant nodules can invade the extracapsular tissues through these structures (6); however, this is unlike benign tumors which cannot invade extracapsularly. The closer the malignant nodules to the capsular, the more likely the malignant tumors are to invade the extracapsular site (7). The invasion and metastasis process requires the accompaniment and formation of neo-angiogenic microvessels. The blood vessels needed to supply malignant tumors can rapidly grow under the action of various

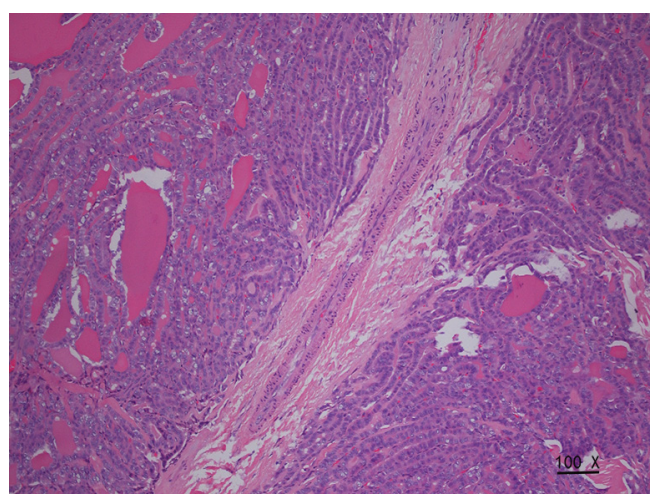

Figure 3 Pathological characteristics of extracapsular vascular invasion (HE staining). The neovascularization of nodules crossed the thyroid capsule and invaded the extrathyroid tissue.

regulatory molecules such as vascular endothelial growth factor, which provides a basis for tumor metastasis and invasion (8).

A vascular invasion has been identified as an essential and independent prognosticator in many cancers $(9,10)$. However, vascular invasions in thyroid cancer are only currently defined pathologically. This study aimed to determine the ultrasonographic signs of neovascularization in malignant tumors to predict the extracapsular invasion likelihood of tumors, using the advantages of a highfrequency ultrasound combined with the pathological findings. A recent pathological study suggested that invasive blood vessels for malignant tumors are located within the 
tumor envelope or outside the tumor rather than inside the tumor itself (10-12). However, in this study, we observed that there are two types of blood flow in the thyroid nodules using a color Doppler. One is non-extracapsular invasive blood flow; that is, blood flow signals could be observed inside or around thyroid nodules; however, this blood flow did not extend outside the capsule. The other type of extracapsular invasion was when the blood flow signals could be observed inside or around the nodules. The blood flow extended across the capsule to the outside of the gland. Furthermore, the incidence rate of malignant nodules was significantly different in nodules within the two distinct types of blood flow. More importantly, we reported that the incidence rate of ETE in the nodules with extracapsular vascular invasion was significantly higher, indicating that extracapsular vascular invasion can not only be used as a biomarker of benign and malignant nodules but as a good indicator of ETE in malignant nodules.

In this study, we found that benign and malignant nodules were shown to have significant differences in the maximum diameter of the nodules, internal microcalcification, irregular nodule edges, aspect ratio, internal blood flow, and the presentation of bilateral cervical lymph nodes. Microcalcification, irregular edges, and aspect ratios $>1$ are the most specific indicators for judging malignant nodules, according to the 2015 American Thyroid Association management guidelines for adult patients with thyroid nodules and differentiated thyroid cancer (11). Our study found that the distribution of blood flow types also showed a significant difference between the two groups in addition to the above indicators, which provides a new method for evaluating benign and malignant nodules $(\mathrm{P}<0.001)$.

The thyroid gland is covered by two layers of connective tissues. The inner layer is a fibrous capsule, a true envelope of the thyroid gland, which is also referenced as the true capsule in the clinic. It envelops the thyroid tissues and penetrates the parenchyma of the gland, dividing the gland into several small thyroidal lobes of various sizes. The outer envelope includes the anterior tracheal fascia from the deep neck fascia. The pseudo capsule is usually absent in the anterior thyroid gland, which allows the glandular tissues of the thyroid isthmus to be directly connected to the extra-thyroid tissue and even the muscle tissues. The thyroid isthmus is usually not enveloped by the membrane, which allows malignant nodules located in the isthmus to have more direct contact with the extrathyroidal tissue, consequently increasing the likelihood of ETE compared with nodules located inside the thyroidal lobes (13). Therefore, for the malignant nodules found in the isthmus, the presence of extravascular capsule invasion must be given more considerable attention.

Furthermore, because the thyroid isthmus usually has no envelope tissue wrapping, it is difficult to judge the relationship between the capsule and the blood flow. This study did not include the isthmic nodules in the study considering the above reasons. For nodules located outside the isthmus, the signs of extracapsular vascular invasion can be well recognized, which is essential for determining if the nodules are benign or malignant and whether ETE occurs.

Due to the lack of a complete capsule in the thyroid gland, adequate ultrasonographic indicators for the prediction of extracapsular invasion are currently lacking. Kamaya et al. have previously shown that the degree of contact between the nodule and the capsule (1). They determined that the length of the contact is between the capsule and the perimeter of the nodule. Furthermore, the study also found some valuable indicators for predicting extracapsular invasion, such as the bulging of the thyroid capsule contour and echo disruption. Furthermore, it is essential to note that determining these indicators mainly relies on the examiner's subjective visual judgment, making standardization challenging. Extracapsular vascular invasion is a useful evaluation index that can be easily observed and measured. It is a clear sign of color Doppler imaging, revealing the invasion effects of the malignant tumors on the capsule by clarifying the relationships between the thyroid capsule and the tumor blood vessels. Compared with indicators that require visual observation, it is easier to repeat and more objective. However, not all sub-mucosal malignant nodules showed extracapsular vascular invasion ability. In this study, out of the 84 malignant nodules, 78 nodules showed extracapsular vascular invasion ability $(92.86 \%)$. It is worth noting that this study does not include nodules without blood flow signals. Therefore, the sole reliability of extracapsular vascular invasion as a predictor of ETE is likely not sufficient; instead, for malignant nodules that are close to the capsule, it is necessary to evaluate and predict based on several indicators.

Jeon et al. found that blood vessel thickness inside and outside of the thyroid gland holds a predictive value for determining ETE (5). However, the measurement of blood vessel thickness by different physicians often produces high variability and does not yield good reproducibility as a metric. In this study, we determined that sufficient couplants should be applied to the probe, and the pressure 
on the blood vessels and nodules should be reduced to improve the display rate of extracapsular vascular invasion during the scanning process. Multiple sections were scanned in series to avoid a blind spot for the subcapsular nodules with high suspicion of ETE. For nodules rich in blood flow, it was necessary to adjust the appropriate color gains, sampling volumes, and speed scales to reduce the disturbances caused by the blood flow that surrounds the outside of the capsule.

The limitation of this study is that as a single institution study, there may be bias in choice. In the future, there is a need to expand this single-institution study further to deter selection bias. And the case samples were too small. Also, since the ability of ultrasound to display posterior and lateral thyroid capsules is limited, additional tools may be considered to extend the coverage to include nodules in contact with the thyroid posterior or lateral capsules.

\section{Conclusions}

We conclude that high-frequency ultrasound can indirectly reflect the interactions between the capsule and the thyroid tumor via a relationship between the vessels of the thyroid tumor and the capsule. This relationship allows for the characterization of the tumor class and provides a useful indicator for predicting ETE.

\section{Acknowledgments}

Funding: This study was supported by the following government funds: (I) Natural Science Funds of Fujian (2017J01289); (II) Medical Innovation Projiect of Fujian (2017-CX-32); (III) National Natural Science Foundation of China (81600669).

\section{Footnote}

Reporting Checklist: The authors have completed the STROBE reporting checklist. Available at http://dx.doi. org/10.21037/tcr-20-888

Data Sharing Statement: Available at http://dx.doi. org/10.21037/tcr-20-888

Conflicts of Interest: All authors have completed the ICMJE uniform disclosure form (available at http://dx.doi. org/10.21037/tcr-20-888). The authors have no conflicts of interest to declare.
Ethical Statement: The authors are accountable for all aspects of the work in ensuring that questions related to the accuracy or integrity of any part of the work are appropriately investigated and resolved. The study was conducted in accordance with the Declaration of Helsinki (as revised in 2013). This retrospective study was approved by the Ethics Review Committee of the First Affiliated Hospital of Fujian Medical University \{[2016]117\}. The review board determined that informed consent was not needed from the enrolled patients.

Open Access Statement: This is an Open Access article distributed in accordance with the Creative Commons Attribution-NonCommercial-NoDerivs 4.0 International License (CC BY-NC-ND 4.0), which permits the noncommercial replication and distribution of the article with the strict proviso that no changes or edits are made and the original work is properly cited (including links to both the formal publication through the relevant DOI and the license). See: https://creativecommons.org/licenses/by-nc-nd/4.0/.

\section{References}

1. Kamaya A, Tahvildari AM, Patel BN, et al. Sonographic Detection of Extracapsular Extension in Papillary Thyroid Cancer. J Ultrasound Med 2015;34:2225-30.

2. Kim SY, Kwak JY, Kim EK, et al. Association of Preoperative US Features and Recurrence in Patients with Classic Papillary Thyroid Carcinoma. Radiology 2015;277:574-83.

3. Kwak JY, Kim EK, Youk JH, et al. Extrathyroid extension of well-differentiated papillary thyroid microcarcinoma on US. Thyroid 2008;18:609-14.

4. Tran B, Roshan D, Abraham E, et al. An Analysis of The American Joint Committee on Cancer 8th Edition T Staging System for Papillary Thyroid Carcinoma. J Clin Endocrinol Metab 2018;103:2199-206.

5. Jeon HM, Lim BJ, Chang HS, et al. The definition of minimal extrathyroid extension in thyroid pathology by analyzing sizable intra- and extrathyroid blood vessels. Korean J Pathol 2012;46:548-53.

6. Mete O, Rotstein L, Asa SL. Controversies in thyroid pathology: thyroid capsule invasion and extrathyroidal extension. Ann Surg Oncol 2010;17:386-91.

7. Wang QC, Cheng W, Wen X, et al. Shorter distance between the nodule and capsule has greater risk of cervical lymph node metastasis in papillary thyroid carcinoma. Asian Pac J Cancer Prev 2014;15:855-60. 
8. Ruoslahti E, Rajotte D. An address system in the vasculature of normal tissues and tumors. Annu Rev Immunol 2000;18:813-27.

9. O'Neill CJ, Vaughan L, Learoyd DL, et al. Management of follicular thyroid carcinoma should be individualised based on degree of capsular and vascular invasion. Eur J Surg Oncol 2011;37:181-5.

10. Mete O, Asa SL. Pathological definition and clinical significance of vascular invasion in thyroid carcinomas of follicular epithelial derivation. Mod Pathol 2011;24:1545-52.

11. Haugen BR, Alexander EK, Bible KC, et al. 2015 American Thyroid Association Management Guidelines for Adult

Cite this article as: Lin M, Su Y, Huang Y, Zhang H, Zeng J, Zhuang Y, Ye X, Chen S. Ultrasonographic evaluation of extracapsular vascular invasion for subcapsular nodules of the thyroid. Transl Cancer Res 2020;9(10):6464-6470. doi: 10.21037/ tcr-20-888
Patients with Thyroid Nodules and Differentiated Thyroid Cancer: The American Thyroid Association Guidelines Task Force on Thyroid Nodules and Differentiated Thyroid Cancer. Thyroid 2016;26:1-133.

12. Collini P, Sampietro G, Pilotti S. Extensive vascular invasion is a marker of risk of relapse in encapsulated nonHürthle cell follicular carcinoma of the thyroid gland: a clinicopathological study of 18 consecutive cases from a single institution with a 11-year median follow-up. Histopathology 2004;44:35-9.

13. Lee YS, Jeong JJ, Nam KH, et al. Papillary carcinoma located in the thyroid isthmus. World J Surg 2010;34:36-9. 